\title{
Rounding off of machine-cutting blades made of sintered carbides
}

\section{Zaokrąglanie krawędzi skrawających ostrzy z węglików spiekanych}

\author{
PIOTR CICHOSZ \\ MIKOLAJ KUZINOVSKI \\ MITE TOMOV \\ ADAM URYCH *
}

The paper describes the origins of edge rounding of machine-cutting blades made of sintered carbides. Various processing machines and abrasive materials used to smoothen the cutting edges are presented. Various methods of measurement of the edge radius are compared. Stressed are the possibilities of improvement of tool durability through the use of new finishing process technologies for tool working surfaces.

KEYWORDS: machine cutting, carbide tools, cutting edge radius, machine tools

Until recently, it was believed that the sharper the edge of the tool, the better the cutting properties. The idea of rounding the cutting edges of carbide blades probably arose quite accidentally during attempts to improve the exploitation properties of drills [5]. When making holes, especially deep ones, it was always difficult to remove chips from the cutting zone. This has been dealt with in various ways, such as: periodic retraction of the tool from the bore, internal feeding of high-pressure machining fluids and the use of lubricating coatings based on lubricants - e.g. molybdenum disulfide or graphite [2]. These coatings wiped fairly quickly due to the relatively low hardness and low temperature resistance, they were finally given up, and their lubrication functions were replaced by polishing chip flutes, which also effectively facilitated chip evacuation. During the lathing of the chip groove, the cutting edges became rounded, which was an unintentional effect. It turned out, however, that this did not deteriorate the cutting properties of the tool, and even caused a significant increase in its durability. The rounding of the cutting edges with abrasive methods has therefore begun to be used in other tools, e.g. in end milling cutters or multi-blade cutting inserts.

It should be noted that the rounding of the edges or even the introduction of protective sheaves on the surface, known as protective chamfers, have long been used in blades from very hard and brittle materials, such as ceramics and regular boron nitride. In these cases, however, it is about eliminating tensile stresses in the blade, which can lead to edge breaking.

\footnotetext{
* Prof. dr hab. inż. Piotr Cichosz (piotr.cichosz@pwr.edu.pl) - Katedra Obrabiarek i Technologii Mechanicznych, Wydział Mechaniczny Politechniki Wrocławskiej; prof. dr inż. Mikolaj Kuzinovski (mikolaj.kuzinovski@ mf.edu.mk), assoc. prof. Mite Tomov, PhD (mite.tomov@mf.edu.mk) - Wydział Mechaniczny Uniwersytetu w Skopje; mgr inż. Adam Urych (adam.urych@kometurpol.pl) - KOMET URPOL
}

Rounding the cutting edges is often incorrectly called honing. In Polish, honing, called more honing, is the process of surface forming with abrasive sticks fixed in heads performing precisely defined kinematic motions, with controlled pressure on the machined surfaces. Such a process has never been used to shape cutting tools, and especially their edges.

The rounding of the cutting edges of the tools to which the anti-wear coatings are then applied has another task: to ensure better adhesion of the coating to the substrate and to reduce its tendency to peel off in the most critical places, such as the edge of the blades.

\section{Machine tools for polishing and rounding edges}

There are many design solutions for machine tools - for smoothing, polishing, deburring, edge rounding or surface cleaning - which use abrasive methods [15]. These processes are often assisted by chemical digestion. Usually, reels containing loose abrasive shapes, abrasive and shaped objects are loaded into the machining drums of the sandblaster. Appropriate drum motions cause the components of the batch to rotate and move relative to each other. In this way, the surface is treated, including the edges of the objects.

In the case of shaping tools with very hard, sharp edges in dressing tools, there is a high risk of mechanical damage to tools that collide with each other. The tools to be machined must therefore be separated from each other and fixed in such a way that they do not damage each other or hit the walls of the container. Many technologies and modified machine tools have been developed to enable such machining.

Figure 1 shows a container smoothing machine with a non-rotating container. The container only lifts up, which causes the tools to submerge in the batch and allows their processing. After the process is completed, the container is lowered to replace the batch of tools to be processed. The smoothing machine is made of a main spindle, on which four rotating heads with planetary motion are mounted on the satellite (fig. 2). Each head has five handles rotating relative to its own axis. It is possible to set the tool spindles at an angle of $0 \div 20^{\circ}$ from the vertical. The inclination of the tools is important due to the risk of clogging by the abrasive channels of the machining media into the cutting zone. If this phenomenon occurs, then after the smoothing operation, the channels should be unblocked, e.g. with the 
method of blowing with compressed air or transferring liquid/hot water vapor. The machine tool allows to round the edges of up to 20 tools in one operation. There are also extractor, for example Rösler, even equipped in 12 spindles, in which shaped objects can be attached.

a)

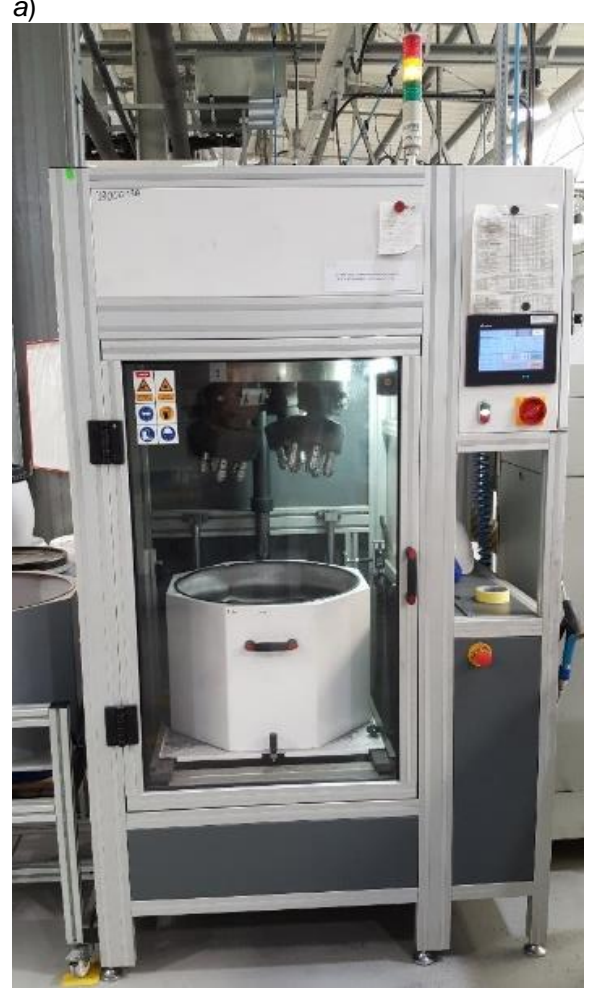

Fig. 1. PARDUS $6 \mathrm{H}$ 30T $2 \mathrm{~S}$ container windrower:

a) general view, b) mounting heads with visible one mounted tool
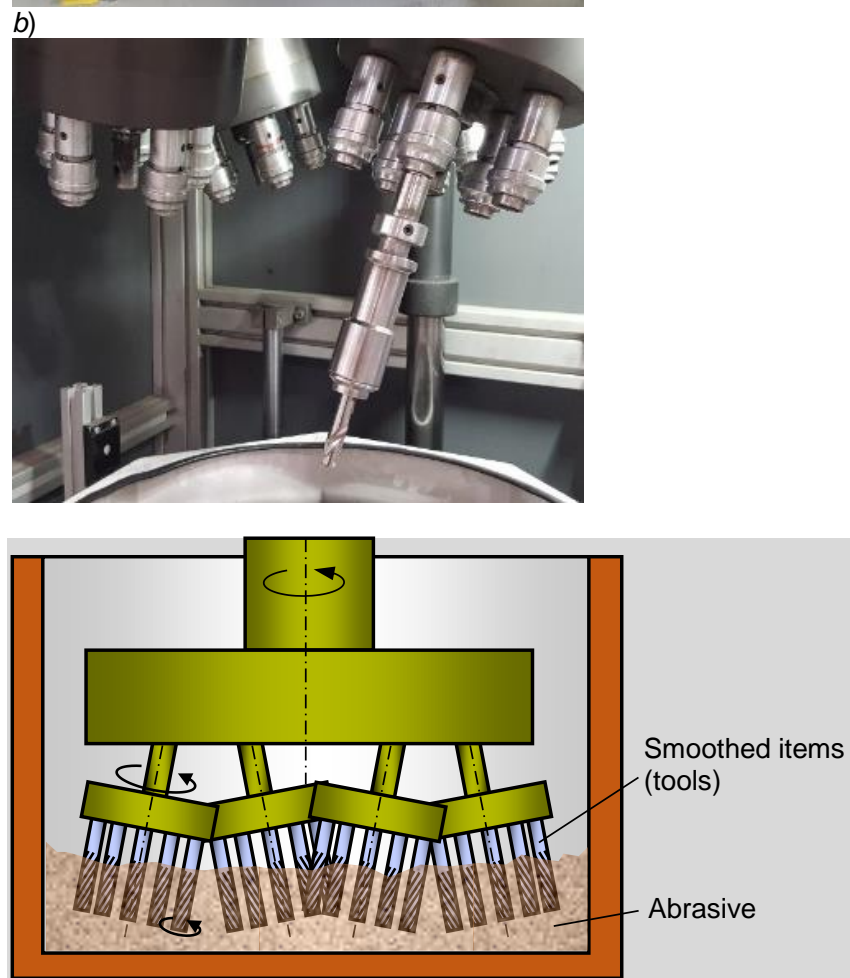

Fig. 2. Diagram of rotary movements of PARDUS $6 \mathrm{H} 30 \mathrm{~T} 2 \mathrm{~S}$ container smoothing spindles

After starting the machine, the main spindle and the rotary heads start to rotate at a small speed, and the tank slowly rises to get the right depth of immersion of the tools in the abrasive stock (fig. 2). From this moment, the machine measures the machining time and changes the direction and time of rotation in a programmed way. The rotary motion causes strong friction between the tools and the abrasive.

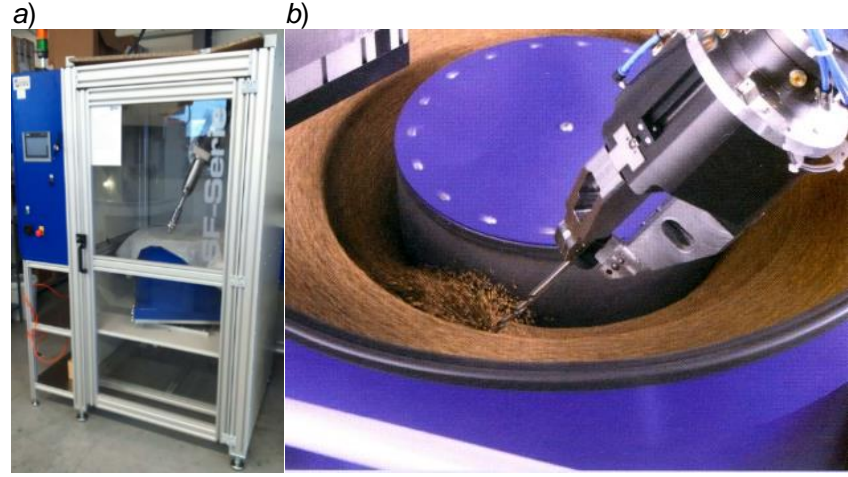

Fig. 3. SF-1-68-M-D machine tool: a) general view, b) the appearance of the container with the shaped tool

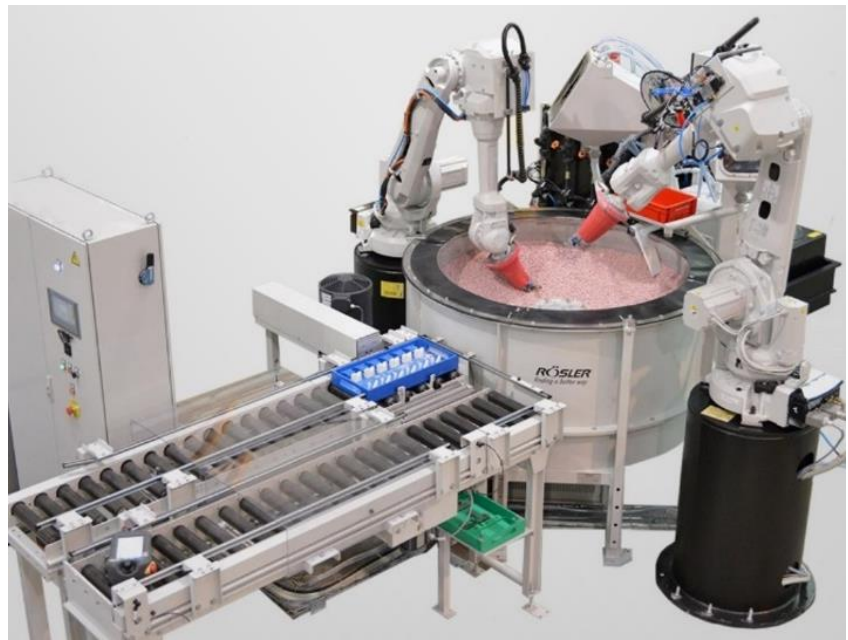

Fig. 4. Rösler container shaper

With this machine (and most others), the final machining effect can be influenced by:

- type of abrasive;

- rotational speed of the main spindle on which the heads are mounted;

- rotational speed of heads;

- direction, time and types of spindle rotation cycles;

- the depth of immersion of tools in the batch;

- tilt angle of the heads.

Figure 3 shows a container shredder with a rotating container for machining cutting tools and other objects. This type of smoothing machine is sometimes called - not very accurately - smoothing machines or jet polishers [8, 15]. The drum rotating at high speed maintains the abrasive load - thanks to the centrifugal force - at the outer walls of the container. At the same time, thanks to this force, the consistency of the batch becomes more compact and affects abrasively on the tools placed in it with greater intensity, which reduces the processing time. Tools are mounted in spindle mounts that can rotate at adjustable speed and in both directions. Changing the angle of spindles allows you to obtain different profiles of rounding edges (Fig. 3). The relatively high rotational speed of the container means that the speed of impact of the batch on the tool reaches even several meters per second. Other container smoothing machines do not provide such an intensive treatment. The disadvantage of these smoothing machines is the relatively small number (up to five pieces) of tools that can be shaped at the same time.

Figure 4 shows an automated post-tray smoothing machine equipped with two robots for placing workpieces in a rotating drum.

An unquestionable advantage of such a stand is quite a large, maintenance-free operation. Robotic arms pick and place workpieces themselves according to pre-programmed cycles. 
a)

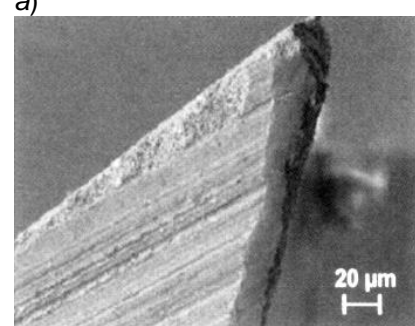

b)

Fig. 5. Cutter blade: a) before processing, $b$ ) after machining in a container smoothing machine with a rotating drum (according to OTEC)

Figure 5 shows the corner of the cutter blade before processing and after shaping in a container shredder with a rotating drum.

Another method that can be used to round the cutting edges is the micro-anemic treatment (fig. 6). Its essence is that the magnetic field lines hold the particles of the magnetic-abrasive conglomerate, and as a result of the complex oscillatory and rotational movements of the tool there is a delicate abrasive effect on its surfaces and edges.

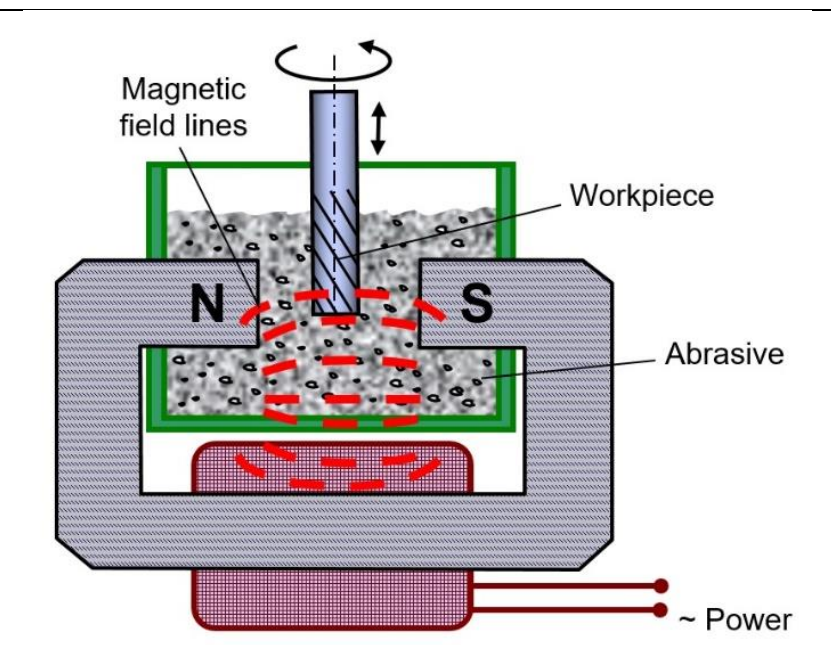

Fig. 6. Magnetic-abrasive treatment in a permanent magnetic field

The disadvantage of this method is the necessity of using specific abrasives, i.e. particles from a binder with magnetic properties with embedded abrasive grains, most often diamond. The worn tool material usually has magnetic properties, which can cause abrasive impurities difficult to separate.

Abrasive blasting (referred to as sandblasting - fig. 7) with precisely controlled parameters - such as: medium pressure, type of abrasive and direction of its application, and processing time - is particularly suitable for rounding the edges of cutting inserts.

There are two varieties of sandblasting - wet and dry. In the first case, abrasive grains move in the liquid stream and in the second - in the air stream.

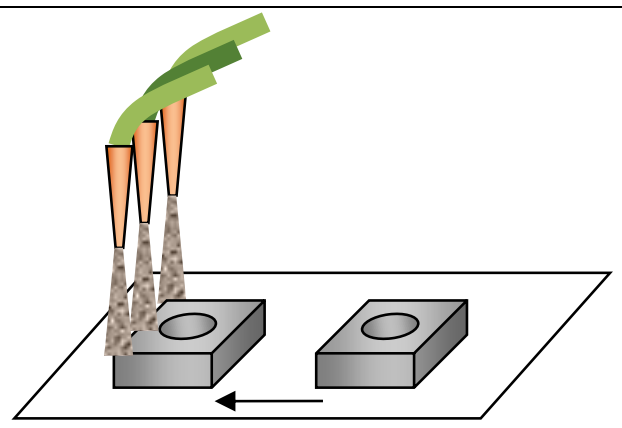

Fig. 7. Diagram of sanding of cutting inserts
Due to the wetting of abrasive grains, the wet method is often more efficient, but also more expensive.

Brushing or polishing with polishing wheels or brushes can be done using robots or by hand. In the case of the production of cutting tools on multi-axis grinders, the brush can be mounted like a grinding wheel, due to which the brushing process takes place directly after grinding on the same machine, it is precise and repeatable. An example of an abrasive brush intended for polishing surfaces and working edges of tools is shown in fig. 8 .

By these methods, the most often smoothing/polishing of the working surfaces of the blades and chip flutes (also after application of protective coatings) and rounds the cutting edges, is made.

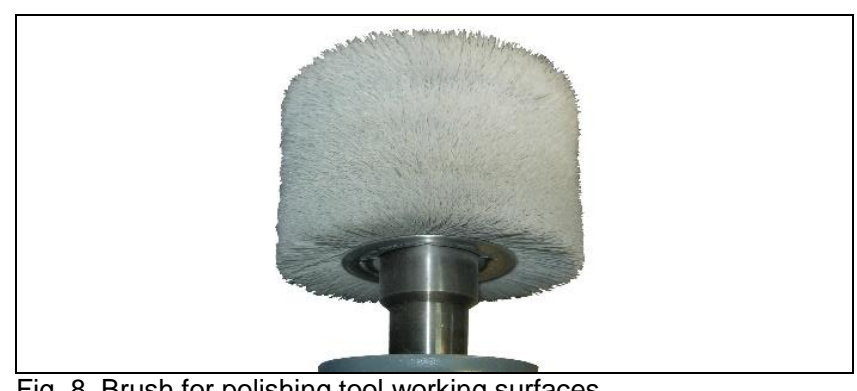

Fig. 8. Brush for polishing tool working surfaces

\section{Types of abrasives}

Abrasives are found in many varieties and have a variety of graininess. The use of a particular product depends on the nature of the process and the expected results of the abrasive treatment.

If the high machining efficiency is a priority, and the roughness of the surfaces and edges being machined is less important, then grains with sharp, hard edges with classic abrasives can be used as the abrasive. At the other extreme are abrasives with a very subtle influence, e.g. glass beads with diameters on the order of tenths of a millimeter, used for gentle deburring and polishing of surfaces in abrasive blasting, and even fine rubber balls with embedded diamond powder. Organic abrasives derived from ground nut shells or seeds are also used, as well as even more delicate materials, e.g. from ground corn cobs. Organic materials should be impregnated from time to time so that they do not deteriorate in a humid work environment. Organic abrasives belong to the least efficient volumetric materials, but they provide exceptionally smooth surfaces.

The abrasive can be: loosely applied in a stream of air or water, loosely fixed in a polishing disc or maintained by magnetic force lines or by centrifugal and gravitational force in vibrating windrower drums. In addition, it can be a component of the abrasive fibers of brushes. Almost every edge milling machine manufacturer also offers abrasive media suitable for the application. Before machining, the cutting tools must be thoroughly cleaned and degreased to ensure repetitive machining results and to prevent contamination of the abrasive medium (especially when the machining is carried out dry).

\section{Measurement of edge rounding}

It should be remembered that the anti-wear coating applied to the cutting blades increases the radius of the rounding of their edges. This change can be significant, although the thickness of typical protective coatings is usually a few micrometers. The optimal radius of edge rounding depends on the purpose of the tool and can range from several to several dozen micrometers. Measurement of this size should not be burdened with too much error to be able to precisely control the final phases of the technological process of smoothing the cutting edges of tools. 
There are many ways to measure the radius of the edge rounding. The simplest is the use of a contact profilometer. A normalized measuring needle usually has an apex angle of approx. $90^{\circ}$ and a radius of apex rounding of $2 \mu \mathrm{m}$. This disrupts the measurement even when the direction of needle travel is set with high precision, perpendicular to the bisecting angle of the $\beta \mathrm{n}$ blade. In this method, from the measured value of the radius of the rounding edge $\mathrm{rn}$, the radius value of the tip of the measuring needle, which replicates on the obtained profilogram, should be subtracted.

Another method of measuring the radius $r_{n}$ is to make a metallographic report perpendicular to the edge of the blade and observe it at high magnification. Among the disadvantages of this method can be mentioned destructive nature and time-consuming, as well as the fact that due to the severity of the edge it is not known exactly where the nicks are made. This may result in a fairly large spread of measurement results, while the execution of several dozen deaths to average the obtained values is very troublesome.

Optical methods are usually used to measure the edge rounding, in which the shadow bars are projected - usually at an angle of $45^{\circ}$ - to the cutting edge. Depending on the shape of the surface, the bands bend, which can be observed under a microscope. This method has been known for a very long time. She was accused of her time, but now, in the era of rapid numerical analysis of images, she has returned to favor. Microscopes allowing this type of measurements can be expensive - the price depends on the accuracy, speed of operation, the degree of activity automation, visualization and data archiving.

Another optical method used to map 3D geometry of the blade is focal differential microscopy [14]. It consists in the placement of only pictures of sharp images from successive layers of the stack of images recorded at various levels. Thanks to this, it is possible to reproduce very precisely the topography of surfaces with relatively large differences in the depth of outlines, including those with large inclination angles. The registered image can undergo a detailed numerical analysis, including determination of roughness parameters along selected directions.

An example of observation and measurement of the radius of edge rounding using the Alicon microscope is shown in fig. 9. The measurements along the cutting edge are carried out automatically, e.g. in 50 cross-sections, and the result $r_{\mathrm{n}}$ is given as an average value. A large number of measurements, on the basis of which the average value $r n$ is determined, is important because the radius is usually determined taking into account about $1 / 4$ of the circle circumference. Considering the surface mapping error, the jaggedness of the edges and the small value of the radius itself (often, for example, several micrometers), the uncertainty of a single measurement may be significant.

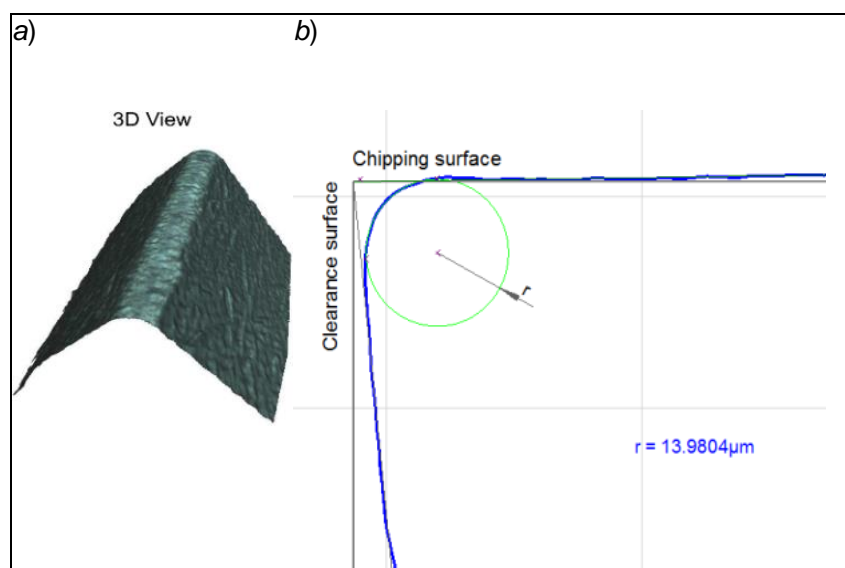

Fig. 9. Measurement of the rounding radius of the cutting edge: a) view of the cutting edge, b) graphic representation of the edge section
A more advanced optical method is a confocal microscope, which has several varieties. In simplification, it consists in the fact that in the optical path between the examined object and the image recording matrix there are very small diameter diaphragms, placed on solid or rotating discs, used to limit the light fragments of the image. Appropriately formed and reflected from the examined object, the light spot returns through a similar shaping system (including the iris) and goes to the measuring matrix. The optical system separates the scattered light, especially those coming from other levels, from outside the plane of sharpness. The position of the focus plane is also very precisely recorded and the image fragments located at this level are assigned to it. Thanks to this, you can receive 3D images with good contrast and large depth of field, even at very high magnification. Registered numerical edge image can - as in the previous method - undergo any graphical analysis.

In order to eliminate the need to use expensive microscopes to measure the radii of rounding of the cutting edges, you can examine slightly different indexes characterizing this rounding. These may be, for example, the extent of the $S_{\alpha}$ or $S_{\gamma}$ edge contraction, relative to its original outline, measured respectively along the relief or attack surface (fig. 10). These indices and $\Delta r$ determine the size of the edge rounding, while $K=S_{\gamma} / S_{\alpha}$ is a measure of its asymmetry, which can significantly affect the durability of the tool. It is enough to even measure an ordinary workshop microscope to measure these indicators.

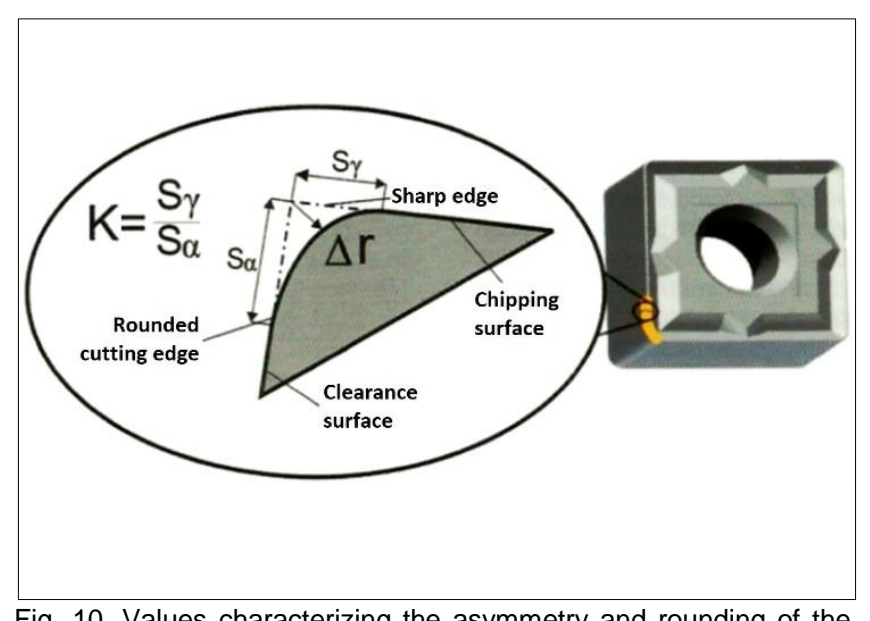

Fig. 10. Values characterizing the asymmetry and rounding of the edges [8]

\section{Conclusions}

The operational properties of the tools can be clearly improved by rounding the cutting edges in the abrasive machining process (smoothing and polishing). Such a method of final forming of the blades results in even several times extending tool life $[1,2,5-8]$. This favorable phenomenon is explained, among others obtaining:

- significantly lower roughness of the working surfaces of the blade and thus less friction on the surfaces of the tool contact with the workpiece;

- less edge jaggedness, which consequently reduces the abrasive wear and the adhesive edge;

- better adhesion of protective coatings in the most sensitive location of the blade, i.e. on the cutting edge; - greater resistance to impact spalling of the coating from the cutting edge.

These benefits have resulted in the fact that companies producing and regenerating tools are increasingly using edge rounding. There are many solutions of machine tools or devices used for this purpose. They differ in the degree of mechanization and automation, but usually allow programming the course of the entire technological process, 
taking into account the direction and value of rotation, depth and place of immersion of the tools in the abrasive charge, the duration of individual stages of the cycle, etc. Some devices allow for very high efficiency due to the option simultaneous machining of many tools and intensification of the impact of abrasive.

Due to the complex structure of some cutting tools, there are two approaches to the mechanization of the operation of rounding the edges of the blades. On one hand, the aim is to fully automate and control this process in order to achieve high repeatability of the machining results. On the other hand, the complexity of cutting tools can hinder the automation of their machining, and sometimes even enforce manual processing, the effects of which depend on the skill and experience of the operators.

It is worth mentioning that the smoothing machines for rounding the cutting edges can also be used to polish the remaining surfaces of the tool, to give them a more aesthetic, mirror-like appearance that facilitates maintenance and attracts the attention of potential buyers.

\section{REFERENCES}

1. S. Baczyński S. „Automatyzacja stanowiska do zaokraglania krawędzi polerowaniem rotacyjnym wraz z doborem parametrów obróbki”. Praca dyplomowa inżynierska, Wydział Mechaniczny, Politechnika Wrocławska, Wrocław 2017.

2. Cichosz P. „Narzędzia skrawające”. Warszawa: WNT, 2006.

3. Carlos J.C.R. "Cutting edge preparation of precision cutting tools by applying micro-abrasive jet machining and brushing". Praca doktorska, Unidruckerei der Universität Kassel, Kassel 2009.

4. Informational materials of the Alicona company.

5. Informational materials of the Gühring company.

6. Informational materials of the Komet company.

7. Informational materials of the MARBAD company.

8. Informational materials of the OTEC company.

9. Informational materials of the PARADUS company.

10. Informational materials of the Rösler company.

11. Informational materials of the Zoller company.

12. Marciniak M., Stefko A., Szyrle W. „Podstawy obróbki w wygładzarkach pojemnikowych". Warszawa: WNT, 1983.

13. Uhlmann E., Oberschmidt D., Kuche Y. „Zautomatyzowana kontrola krawędzi skrawających narzędzi”. MM Magazyn Przemysłowy. 4, 146 (2015).

14. Wieczorowski M. „Metrologia nierówności powierzchni Metody i systemy". Skórzewo: ITA, 2013.

15. Woźniak K. „Obróbka powierzchni w wygładzarkach pojemnikowych". Warszawa: WNT, 2017.

Translation of scientific articles, their computer composition and publishing them on the website www.mechanik.media.pl by original articles in Polish is a task financed from the funds of the Ministry of Science and Higher Education designated for dissemination of science. 